\title{
Modern Diagnosis in the Evaluation of Pulmonary Vascular Disease
}

\author{
Alexander A. Bankier and Carole Dennie
}

\section{Learning Objectives}

- To provide clinical background information about the most relevant vascular disorders of the thorax

- To elucidate the role of imaging in the work-up of these disorders

- To discuss the role of diagnostic imaging in patient management and clinical decision-making

\subsection{Introduction}

The conventional chest radiograph, computed tomography (CT), and, with restrictions, magnetic resonance imaging (MRI) are the three most commonly used imaging modalities for evaluating patients with suspected pulmonary vascular disease. Additionally, in the very recent past, fluorodeoxyglucose positron emission tomography (FDGPET) and FDG-PET/CT have been attributed clinical usefulness in the evaluation of large vessel vasculitis. In general clinical routine, however, CT remains the imaging modality of choice for evaluation of patients with suspected pulmonary vascular disease. This is currently emphasized by new technical developments, such as dual-source and dual-energy scanners, that enable to simultaneously generate morphological and functional information from a sole data set. Therefore, CT has become crucial for evaluating the pulmonary vasculature. This is based on the following: Among the various imaging modalities available in recent decades, com-

\footnotetext{
A. A. Bankier $(\bowtie)$

Department of Radiology, Beth Israel Deaconess Medical Center, Harvard Medical School, Boston, MA, USA

e-mail: abankier@bidmc.harvard.edu

C. Dennie

Department of Medical Imaging, The Ottawa Hospital, University of Ottawa, Ottawa, ON, Canada

e-mail: Cdennie@toh.ca
}

puted tomography (CT) has remained the core technique for evaluating respiratory disorders. Over the last few years, this central position has been reinforced by the possibility of deriving morphological and functional information from the same data set. This approach is of major interest for evaluating pulmonary vascular diseases for three main reasons: (1) Pulmonary vascular disorders require good morphological evaluation, not only for the vascular tree per se but also the surrounding lung parenchyma; (2) given that we have reached an upper limit in terms of morphologic image resolution, but simultaneously can provide functional information, CT is the ideal combination of high-end morphologic imaging with perfusion and ventilation imaging; and (3) it is becoming increasingly important to evaluate the cardiac consequences or causes of pulmonary vascular diseases, which requires all advantages that $\mathrm{CT}$ can provide. Because of these reasons, and given the general importance of CT, this course will focus on CT. Other imaging modalities will not be disregarded but rather discussed in the case-based presentations and framed in their distinct clinical context.

\subsection{Optimized Evaluation of Pulmonary Vessels on Chest CT Examinations}

\subsubsection{Temporal Resolution of Pulmonary CT Angiograms}

Given the anatomical complexity of the lungs, the most crucial prerequisite for optimal chest imaging is a high spatial resolution, required not only to detect subtle morphologic abnormalities but also to adequately image thoracic vessels on CT angiography (CTA) and to differentiate between normal and abnormal vascular structures and dimensions. However, high spatial resolution is optimally employed when short overall examination times and high temporal resolution (i.e., short acquisition times of the individual axial image planes) are available simultaneously. This allows for 
the analysis of high-resolution images with a minimum of motion artifacts. The technical progress in development of multidetector-row CT (MDCT) has enabled radiologists to image the entire thorax with increasing spatial resolution in decreasing time durations. As a consequence, more patients are able to hold their breath throughout the entire period of data acquisition. This results in a substantial decrease in number and frequency of respiratory motion artifacts. Shorter CT rotation times have furthermore enabled shorter acquisition times and significantly artifacts caused by cardiac motion. These have, in the past, not only decreased image quality in the proximity of cardiac structures but could also cause pulsation artifacts at the anatomical level of systemic and pulmonary vessels, thus mimicking endovascular abnormalities, such as thrombi or neoplastic tissue. In order to image patients with the highest temporal resolution and shortest examination time, it is preferable to choose the shortest rotation time possible and the highest pitch, defined as the table feed per rotation, divided by the nominal beam width at the isocenter of the scanner. For example, on a 64-slice MDCT using a single X-ray source, rotation times range from 0.30 to $0.40 \mathrm{~s}$, resulting in a temporal resolution per image not better than half the rotation time, and the pitch values usually do not exceed 1.5 [1]. The introduction of dual-source 64-slice MDCT technology offers the possibility of improving the technical requirements for CT. When both available tubes are operated at the same kilovoltage, the temporal resolution of each image is $1 / 4$ of the rotation time, each of the two detectors contributing $90^{\circ}$ of data in parallelray geometry to each image plane [2]. Moreover, the second measurement system of dual-source CT allows for a higher pitch mode than those available with a single-source CT, but without image distortion inside the field of view of the second detector. In a recent study, Tacelli et al. showed that this scanning mode provides $\mathrm{CT}$ angiographic examinations of excellent quality for thoracic applications in routine clinical practice, including those referred for pulmonary vascular diseases such as acute pulmonary embolism (PE) [3].

\subsection{Improved Morphological Evaluation of the Peripheral Pulmonary Vasculature}

With the introduction of MDCT, CTA is now recognized as the reference standard for diagnosing acute PE [4]. The advantages of MDCT over single-slice CT result from the opportunity of scanning the entire volume of the thorax with submillimetric collimation in a very short periods of time, most often under the duration of a single breath-hold, which is particularly useful when evaluating dyspneic patients. These technological advances have improved the evaluation of peripheral pulmonary arteries and the accuracy of CT in the work-up of acute PE. Simultaneously, MDCT allows radiologists to scan patients at a low kilovoltage with two subsequent advantages, in particular, the possibility of reducing the doses of contrast material and reducing the overall radiation dose. This is of particular importance in young female patients who may be exposed to substantial levels of radiation to breast tissue. In the context of acute PE, the latter concern is clinically relevant when keeping in mind the lower prevalence of acute PE, which has dropped from 33\% on angiographic studies to $<20 \%$ on CT/MDCT scans. To date, several studies have investigated the clinical benefits of low-kilovoltage techniques [i.e., $80-100 \mathrm{kV}(\mathrm{p})$ vs 120 $140 \mathrm{kV}(\mathrm{p})]$, the parameters at which most CT angiograms are performed. However, for obvious ethical reasons, these studies were based on the comparative analysis of different populations scanned with single-source CT [5-8]. The limitations of these comparisons include the lack of systematic adjustment for individual patient morphology, cardiac hemodynamics, and potential underlying respiratory disease. With the introduction of dual-source CT, it is now possible to two tubes being set at different kilovoltages. In addition to the opportunity to evaluate lung perfusion, standard CT angiograms can also benefit from this scanning mode, which has been shown to improve the ability to analyze small pulmonary arteries with $80 \mathrm{kV}(\mathrm{p})$ [9].

\subsection{Perfusion Imaging with Dual- Energy CT}

Lung perfusion with dual-energy CT does not reflect blood flow analysis per se, as it represents a measurement at only one time point, but it rather reflects an iodine map of the lung microcirculation at this particular time point. There are numerous parameters known to influence the iodine distribution within pulmonary capillaries. Some are techniquerelated, whereas others are due to the anatomical and/or physiological circumstances under which data acquisition was performed. Dual-energy CT angiographic examinations can be obtained using a scanning protocol similar to that utilized in clinical practice. The acquisitions are acquired from top to bottom of the chest, with an injection protocol similar to that of a standard CT angiogram obtained with single energy on a 64-slice scanner. Two categories of images can be reconstructed. Diagnostic scans correspond to contiguous 1-mm-thick transverse CT scans generated from the raw spiral projection data of tube A and tube $\mathrm{B}$ (60\% from the acquisition with tube A, $40 \%$ from the acquisition with tube B). Lung perfusion scans (i.e., images of the perfused blood volume of the lung parenchyma) are generated after determination of the iodine content of every voxel of the lung parenchyma on the separate $80-$ and $140-\mathrm{kV}(\mathrm{p}$ ) images. They can be rendered as gray-scale or color-coded 
images. All images can be displayed as transverse scans, complemented as needed by coronal and sagittal reformats. Even though a dual-energy acquisition does not correspond to true perfusion imaging, several applications of this pulmonary micro-CTA have been investigated [10].

\subsection{Acute Pulmonary Embolism}

Dual-energy CT can detect endoluminal clots on averaged images of tubes A and B as reliably as can single-source CTA [11]. In a preliminary study, the authors validated the detectability of perfusion defects beyond obstructive clots. Perfusion defects in the adjacent lung parenchyma have the typical perfusion-territorial triangular shape well known from pulmonary angiographic, scintigraphic, and magnetic resonance imaging (MRI) perfusion studies. Dual-energy CTA can help predict perfusion defects without directly identifying peripheral endoluminal clots that may be located in subsegmental or more distal pulmonary arterial branches. Dual-energy CT can help differentiate lung infarction from less specific peripheral lung consolidation.

\subsection{Chronic Thromboembolic Pulmonary Hypertension (CTEPH)}

Dual-energy CTA may depict perfusion defects distal to chronic clots (Fig. 17.1). In a study of 40 patients referred with $\mathrm{PH}$ of whom 14 were diagnosed with CTEPH, the sensitivity of DECT was $100 \%$ with a specificity of $92 \%$ compared to planar VQ [12]. DECT still has limitations with regard to imaging artifacts, but these are expected to improve with technical advancements.
Three vascular characteristics of chronic PE may manifest on dual-energy CT imaging. First, chronic PE causes a mosaic pattern of lung attenuation, characterized by areas of groundglass attenuation, with enlarged vascular segments intermingled with areas of normal lung attenuation and smaller vascular segments (Fig. 17.2). When present, these findings are suggestive of blood flow redistribution, but are not consistently seen on conventional CT in patients with chronic PE. In such patients, dual-energy CT has the potential to detect ground-glass attenuation of vascular origin via high iodine content within the areas of ground-glass attenuation, thus

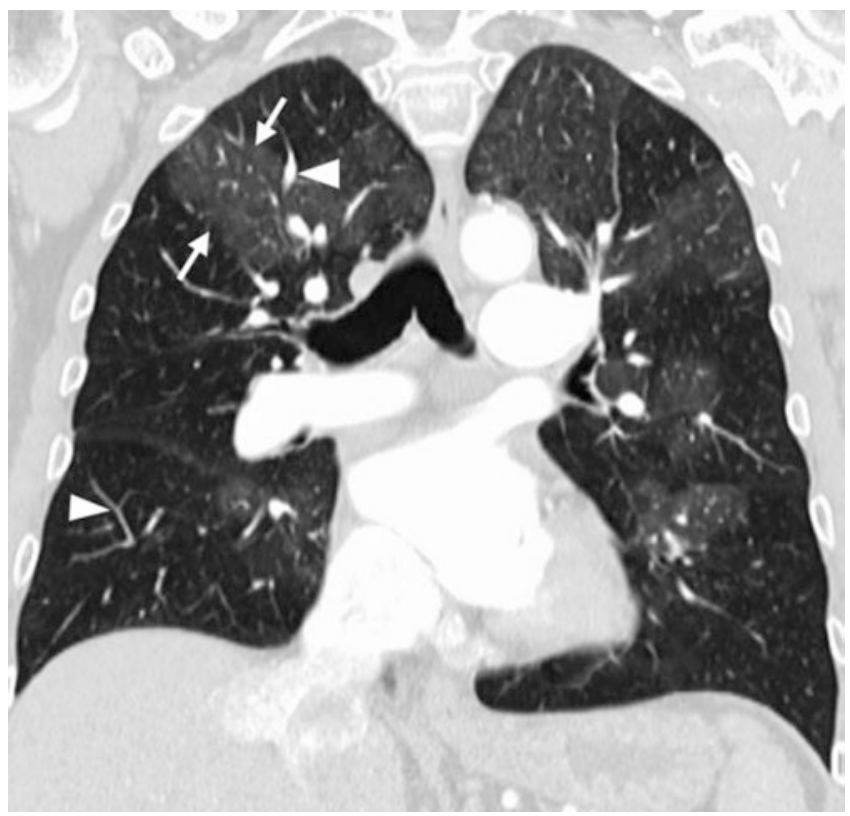

Fig. 17.2 Mosaic attenuation pattern. Coronal reformat (lung window) shows areas of high attenuation (arrows) which contain larger vessels than those within areas of lower attenuation (arrowheads)
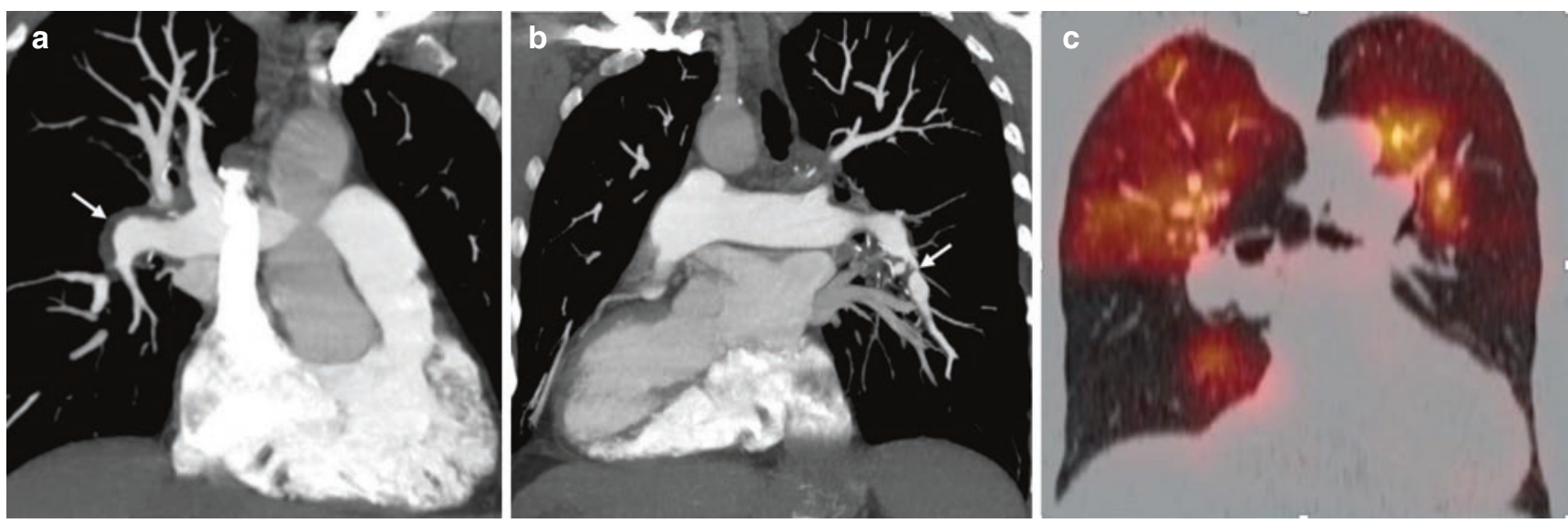

Fig. 17.1 (a-c) Chronic thromboembolic disease. Oblique coronal MIP CTPA image (a) shows a mural filling defect in the right interlobar artery (arrow). Oblique coronal MIP CTPA image (b) in the same patient depicts focal stenosis (arrow) and post-stenotic dilatation involving a segmental artery in the left lower lobe. DECT coronal reformatted image (c) in a different patient reveals almost completely absent perfusion in the lower lobes 


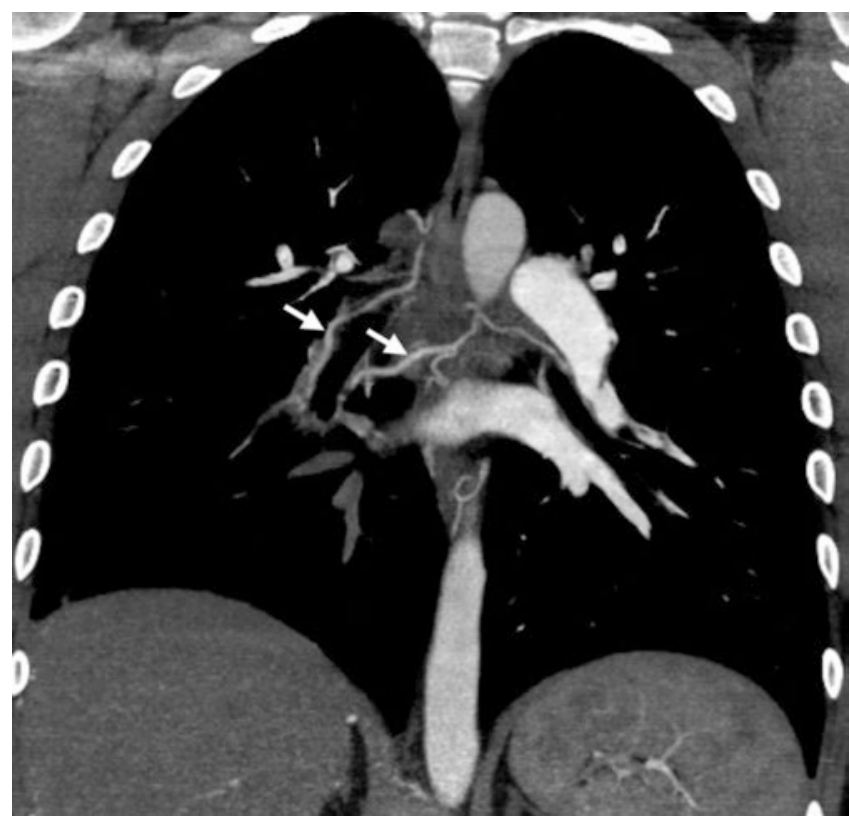

Fig. 17.3 Systemic collateral supply. Coronal reformat CTPA image reveals bronchial artery collaterals (arrows)

enabling their distinction from ground-glass attenuation secondary to bronchial or alveolar diseases [13]. Second, chronic PE can cause calcifications within partially or completely occlusive chronic clots as well as within pulmonary artery walls, when chronic PE is complicated by long-standing or severe pulmonary hypertension. Such calcifications can be detected via virtual non-contrast imaging, accessible by dualenergy CT imaging. Third, the images generated at $80 \mathrm{kV}$ can improve visualization of the systemic collateral supply present in chronic PE that originates from bronchial and nonbronchial systemic arteries (Fig. 17.3).

\section{Key Point}

- With technical advancements, DECT may replace VQ in the future in the detection of chronic pulmonary embolism.

\subsection{Obstructive Airway Diseases}

Abnormalities of pulmonary perfusion are present in numerous smoking-related respiratory diseases. Investigating endothelial dysfunction in pulmonary arteries of patients with mild chronic obstructive pulmonary disease (COPD), Peinado et al. showed that endothelial dysfunction of pulmonary arteries is present even in patients with mild COPD [14]. In these patients, as well as in smokers with normal lung function, some arteries show a thickened intima, suggesting that tobacco consumption may play an important role in the pathogenesis of pulmonary vascular pathologies in COPD. Several structural changes in early stages of COPD have been described in experimental and animal models, including proliferation of smooth muscle fibers within peribronchiolar arterioles and collagen and elastin deposition in the thickened intima of vessels $[15,16]$. In preliminary studies, Hoffman et al. [17] showed an increased heterogeneity of local mean transit times of the contrast material within pulmonary microvasculature of smokers with normal pulmonary function tests. More recently, Pansini et al. studied pulmonary lobar perfusion with dual-source, dual-energy CTA in COPD patients [18]. These authors found that nonsmokers had no alterations in lung structure and that there is a uniform distribution of iodine content within upper and lower lobes and between right and left lungs. Perfusion scans of emphysematous patients showed significantly lower iodine content within lung microcirculation of the upper lobes, as compared to smokers without emphysema, and a significantly lower perfusion in the upper lung zones as compared with lower lung zones, thus matching lung parenchymal destruction. These structural abnormalities are substantial observations, given the epidemiologic and socioeconomic burden of COPD.

\subsection{Restrictive Airway Diseases}

The substantial importance of pulmonary hypertension on the clinical course and prognosis of patients with fibrotic lung disease has been extensively recognized. Similar to obstructive lung disorders, MDCT and DSCT have a role to play in the evaluation of these disorders. Recent research suggests that the mere measurement of pulmonary artery diameters might not be a reliable parameter for disease severity assessment, given the potentially confounding role of parenchymal traction on central and peripheral pulmonary vessels. Moreover, given the age of the population in which these diseases usually occur, age-related changes have to be taken into account of any morphometric-based clinical decision-making and classification. Overall, and despite promising initial scientific evidence, the role of MDCT and DSCT in assessing patients with fibrotic lung diseases still needs to be determined. The many ongoing pharmacological trials, notably in patients with usual interstitial pneumonitis, may provide an ample study ground in this field.

\subsection{Pulmonary Hypertension}

Once left heart causes and intracardiac shunts have been ruled out with echocardiography, CT can play a key role in the classification of patients with pulmonary hypertension 
Table 17.1 Abbreviated Nice classification of pulmonary hypertension

1. Pulmonary arterial hypertension $(\mathrm{PAH})$

a. Idiopathic

b. Heritable

c. Drugs and toxins induced

d. Associated with connective tissue disease, HIV infection, portal hypertension, congenital heart disease, schistosomiasis

- Pulmonary veno-occlusive disease and/or pulmonary capillary hemangiomatosis

- Persistent pulmonary hypertension of the newborn

2. Pulmonary hypertension due to left heart disease

3. Pulmonary hypertension due to lung disease and/or hypoxia

4. Chronic thromboembolic pulmonary hypertension and other pulmonary artery obstructions

5. Pulmonary hypertension with unclear and/or multifactorial mechanisms

(PH) (Table 17.1) [19]. There are vascular, cardiac, and pulmonary parenchymal features of pulmonary hypertension on CT. A main pulmonary artery (MPA) diameter of greater than $29.5 \mathrm{~mm}$ has a sensitivity of $70.8 \%$ and specificity of $79.4 \%$ for the detection of pulmonary hypertension [20]. However, there is no correlation between the degree of $\mathrm{PH}$ and MPA diameter. The specificity is lower in patients who have interstitial fibrosis which is postulated to cause MPA dilatation due to traction without $\mathrm{PH}$ [21]. A ratio of diameter of MPA to that of ascending aorta in the same axial plane of $\geq 1.0$ is also indicative of $\mathrm{PH}$, especially in younger patients $(<50)$, and may be a more useful predictor of $\mathrm{PH}$ in those with advanced interstitial fibrosis [22]. In one study, the segmental artery-to-bronchus diameter ratio of $>1: 1$ in three or four lobes along with a dilated MPA had almost $100 \%$ specificity in the diagnosis of $\mathrm{PH}$ [23].

Other $\mathrm{CT}$ features of PH include right ventricular dilatation and hypertrophy ( $>4 \mathrm{~mm}$ ), straightening or bowing of the interventricular septum, and reflux of contrast into a dilated inferior vena cava \pm hepatic veins. Pulmonary parenchymal features include the mosaic attenuation pattern most commonly seen in patients with chronic thromboembolic pulmonary hypertension (CTEPH). Other less common findings include centrilobular ground-glass nodules due to the presence of cholesterol granulomas, plexogenic arteriopathy or capillary proliferation, and serpiginous centrilobular arterioles as a sign of neovascularity [24].

Electrocardiograph (ECG)-gated MDCT acquisitions of the entire thorax enable the evaluation of novel functional parameters in addition to the standard morphology. In patients with $\mathrm{PH}$, right pulmonary artery distensibility has been reported as an accurate predictor for PH on ECG-gated 64-slice MDCT scans of the chest [25]. In this study, its diagnostic value was superior to that of the single measurement of PA diameter.

\subsection{Right Ventricular Function}

Fast rotation speed and dedicated cardiac reconstruction algorithms designed to extend the conventional multislice acquisition data scheme have opened new opportunities for cardiac and thoracic imaging applications. However, cardiac MRI (CMR) has become the gold standard for the assessment of right function due to superior reproducibility in comparison to echo and its superior temporal resolution compared to CT. CMR also provides prognostic information and can be performed at baseline and in follow-up without the use of ionizing radiation.

Multiple MR parameters have been assessed in patients with PH. Increased right ventricular volumes and reduced stoke volumes at baseline have been shown to be predictors of treatment failure and increased mortality [26]. On velocity-encoded phase contrast imaging, an average velocity in the MPA of $<11.7 \mathrm{~cm} / \mathrm{s}$ has a $92.9 \%$ sensitivity and $82.4 \%$ specificity for the detection of $\mathrm{PH}$, and interventricular septal bowing has been correlated with a systolic pulmonary artery pressure (PAP) of $>67 \mathrm{~mm} \mathrm{Hg} \mathrm{[76].} \mathrm{Swift}$ retrospectively analyzed CMR and right heart catheterization in 233 patients with suspected PH. Ventricular mass index (VMI) was the CMR measurement with the strongest correlation with mean PAP $(r=0.78)$ and the highest diagnostic accuracy (area under ROC $=0.91$ ) for the detection of PH. Late gadolinium enhancement (Fig. 17.4), VMI $\geq 0.4$, and pulmonary artery relative area change $\leq 15 \%$ predicted the presence of $\mathrm{PH}$ with $\geq 94 \%$ positive predictive value [27]. Recently, 4D flow MRI has revealed turbulent vortices

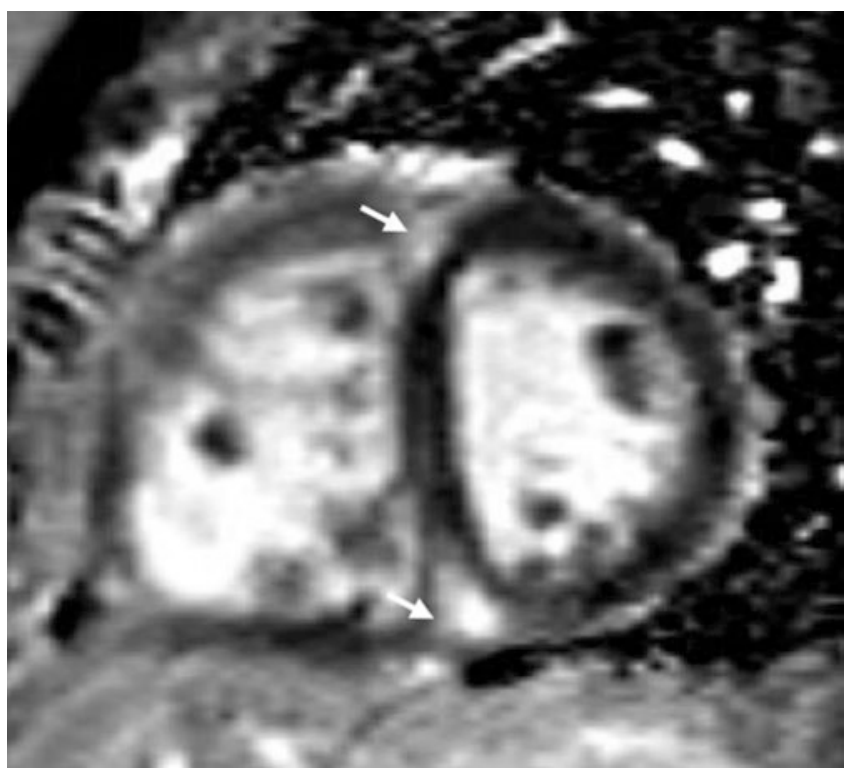

Fig. 17.4 Late gadolinium enhancement short axis image shows the typical enhancement pattern seen in PH with enhancement at the superior and inferior insertion points of the right ventricle into the interventricular septum (arrows) 
in the central pulmonary arteries and has enabled measurement of wall shear stress [28, 29]. Other novel CMR techniques include right ventricular $\mathrm{T} 1$ mapping and strain imaging [30].

\section{Key Point}

- Although CT can be very useful in determining the cause of PH, CMR is the gold standard for the assessment of right ventricular function and can provide information on pulmonary artery dynamics. Importantly, it can also impart prognostic information.

\subsection{Imaging of Pulmonary Vasculitis}

Systemic primary vasculitides are idiopathic diseases causing an inflammatory injury to the vessel walls. Pulmonary involvement is frequent, and chest $\mathrm{CT}$ often in combination with PET/CT is the reference imaging technique in its assessment. Pulmonary vasculitis occurs in a wide variety of systemic and pulmonary vascular disorders. Most vasculitic entities affecting the lung induce overlapping disease patterns such as pneumonitis with or without capillaritis, diffuse alveolar damage and acute pulmonary hemorrhage, or inflammatory obstruction of central pulmonary arteries down to small vessels with chronic secondary pulmonary hypertension with or without interstitial lung disease, and therefore the clinical symptoms per se or the CT morphology alone is often nonspecific.

Owing to their complementary value in imaging of central and peripheral vascular territories and their secondary parenchymal or interstitial abnormalities, CT angiography (CTA), high-resolution CT (HRCT), and fusion imaging play key roles in the noninvasive work-up of patients with suspected pulmonary vasculitis. They enable indicative for further clinical tests, imaging, or invasive diagnostics and direct medical treatment during follow-up. This course will familiarize radiologists with CT diagnostic key features that reflect pulmonary vasculitic pathology inherent to the underlying disease.

\subsection{The Role of CT in the Differential Diagnosis of Pulmonary Vasculitis}

A wide variety of CT pathology of the lung parenchyma, vessels, and airways has been described, and diagnosis is a challenging interdisciplinary field. Clinical and laboratory data have to be included in close cooperation with the refer- ring physician. This course will describe the most common typical and atypical CT features of pulmonary vasculitis and their possible changes over time and therapy, focusing on the differential diagnosis with other inflammatory/infectious hereditary and neoplastic disorders.

In large vessel vasculitis, CT is the method of choice often in combination with PET to discriminate macroscopic vascular abnormality presenting key pathological features such as pulmonary arterial wall thickening with late enhancement, steno-occlusive or thrombo-obliterating disease with resulting oligemia, infarction in the dependent lung, or arterial aneurysms as a facultative cause of massive pulmonary hemorrhage. CT is the modality of choice to demonstrate effects of peripheral small vessel pulmonary vasculitis on the central pulmonary arteries such as secondary chronic pulmonary hypertension due to reduction of the total cross-sectional area with arteriolar remodeling or narrowing of the capillary bed in capillaritis. The variety of peripheral vascular bronchial and parenchymal CT patterns of small vessel vasculitis is great, often complex requiring interpretation in combination with clinical symptoms and laboratory test results. However, many vasculitic disorders present CT features, which are suggestive of a vasculitic disorder. CT-pathologic correlates of the peribronchovascular axial interstitium, pulmonary hemorrhage, types of inflammatory parenchymal infiltration, and secondary pathologies such as organizing pneumonia are discussed with reference to the revised 2012 International Chapel Hill Consensus Conference on pulmonary vasculitis and the American College of Rheumatology (ACR) [31].

\subsection{Concluding Remarks}

Pulmonary vascular diseases are a complex group of conditions with various potential causes. Radiological techniques play a key role in diagnosing and managing these diseases. A thorough knowledge is required from the radiologist about the manifestations of these diseases, and about the reasonable use of the various imaging techniques available, to facilitate the diagnostic process and to streamline patient management.

\section{Take-Home Messages}

- Thorough knowledge of pulmonary vascular diseases is required from radiologists to contribute to diagnosis and work-up.

- The imaging armamentarium plays a key role in the diagnosis and management of these disorders. 


\section{References}

1. Rogalla P, Kloeters C, Hein PA. CT technology overview: 64-slice and beyond. Radiol Clin N Am. 2009;47:1-11.

2. Petersilka M, Bruder H, Krauss B, et al. Technical principles of dual source CT. Eur J Radiol. 2008;68:362-8.

3. Tacelli N, Remy-Jardin M, Flohr T, et al. Dual-source chest CT angiography with high temporal resolution and high pitch modes: evaluation of image quality in 140 patients. Eur Radiol. 2010;20:1188-96.

4. Remy-Jardin M, Pistolesi M, Goodman LR, et al. Management of suspected acute pulmonary embolism in the era of CT angiography: a statement from the Fleischner Society. Radiology. 2007;245:315-29.

5. Holmquist F, Nyman U. Eighty-peak kilovoltage 16-channel multidetector computed tomography and reduced contrast medium doses tailored to body weight to diagnose pulmonary embolism in azotaemic patients. Eur Radiol. 2006;16:1165-76.

6. Sigal-Cinqualbre AB, Hennequin R, Abada H, et al. Low-kilovoltage multi-detector row chest $\mathrm{CT}$ in adults: feasibility and effect on image quality and iodine dose. Radiology. 2004;231:169-74.

7. Schueller-Weidekamm C, Schaefer-Prokop CM, Weber M, et al. CT angiography of pulmonary arteries to detect pulmonary embolism: improvement of vascular enhancement with low kilovoltage settings. Radiology. 2006;241:899-907.

8. Heyer CM, Mohr PS, Lemburg SP, et al. Image quality and radiation exposure at pulmonary $\mathrm{CT}$ angiography with 100- or $120-\mathrm{kVp}$ protocol: prospective randomized study. Radiology. 2007;245:577-83.

9. Gorgos AB, Remy-Jardin M, Duhamel A, et al. Evaluation of peripheral pulmonary arteries at $80 \mathrm{kV}$ and at $140 \mathrm{kV}$ : dual-energy computed tomography assessment in 51 patients. J Comput Assist Tomogr. 2009;33:981-6.

10. Remy-Jardin M, Faivre JB, Pontana F, et al. Thoracic applications of dual energy. Radiol Clin N Am. 2010;48:193-205.

11. Pontana F, Faivre JB, Remy-Jardin M, et al. Lung perfusion with dual-energy multidetector-row CT (MDCT): Feasibilityfor the evaluation of acute pulmonary embolism in 117 consecutive patients. Acad Radiol. 2008;15:1494-504.

12. Dournes G, Verdier D, Montaudon M, et al. Dual-energy CT perfusion and angiography in chronic thromboembolic pulmonary hypertension: diagnostic accuracy and concordance with radionuclide scintigraphy. Eur Radiol. 2014;24(1):42-51.

13. Pontana F, Remy-Jardin M, Duhamel A, et al. Lung perfusion with dual energy multidetector-row CT: can it help recognize ground glass opacities of vascular origin? Acad Radiol. 2010;17:587-94.

14. Peinado VI, Barberà JA, Ramirez J, et al. Endothelial dysfunction in pulmonary arteries of patients with mild COPD. Am J Phys. 1988;274:L908-13.

15. Yamato Y, Sun JP, Churg A, et al. Guinea pig pulmonary hypertension caused by cigarette smoke cannot be explained by capillary bed destruction. J Appl Physiol. 1997;82:1644-53.

16. Santos S, Peinado VI, Ramirez J, et al. Characterization of pulmonary vascular remodelling in smokers and patients with mild COPD. Eur Respir J. 2002;19:632-8.
17. Hoffman EA, Simon BA, McLennan G. A structural and functional assessment of the lung via multidetector-row computed tomography. Proc Am Thorac Soc. 2006;3:519-34.

18. Pansini V, Remy-Jardin M, Faivre JB, et al. Assessment of lobar pulmonary perfusion in COPD patients: preliminary experience with dual-energy CT angiography. Eur Radiol. 2009;19:2834-43.

19. Simonneau G, Gatzoulis MA, Adatia I, et al. Updated clinical classification of pulmonary hypertension. J Am Coll Cardiol. 2013;62:D34-41.

20. McLaughlin VV, Archer SL, Badesch DB, et al. ACCF/AHA 2009 expert consensus document on pulmonary hypertension: a report of the American College of Cardiology Foundation Task Force on Expert Consensus Documents and the American Heart Association developed in collaboration with the American College of Chest Physicians; American Thoracic Society, Inc.; and the Pulmonary Hypertension Association. J Am Coll Cardiol. 2009;53:1573-619.

21. Devaraj A, Wells AJ, Meister MG, et al. The effect of diffuse pulmonary fibrosis on the reliability of CT signs of pulmonary hypertension. Radiology. 2008;249:1042-9.

22. Mahammedi A, Oshmyansky A, Hassoun PM, et al. Pulmonary artery measurements in pulmonary hypertension: the role of computed tomography. J Thorac Imaging. 2013;28(2):96-103.

23. Tan RT, Kuzo R, Goodman LR, et al. Utility of CT scan evaluation for predicting pulmonary hypertension in patients with parenchymal lung disease. Medical College of Wisconsin Lung Transplant Group. Chest. 1998;113(5):1250-6.

24. Peña E, Dennie C, Veinot J, Hernandez Muñiz S. Pulmonary hypertension: how the radiologist can help. Radiographics. 2012;32:9-32.

25. Revel MP, Faivre JB, Remy-Jardin M, et al. Pulmonary hypertension: ECG-gated 64-section CT angiographic evaluation of new functional parameters as diagnostic criteria. Radiology. 2009;250:558-66.

26. Benza R, Biederman R, Murali S, et al. Role of cardiac magnetic resonance imaging in the management of patients with pulmonary arterial hypertension. J Am Coll Cardiol. 2008;52(21):1683-92.

27. Swift AJ, Rajaram S, Condliffe R, Capener D, et al. Diagnostic accuracy of cardiovascular magnetic resonance imaging of right ventricular morphology and function in the assessment of suspected pulmonary hypertension results from the ASPIRE registry. J Cardiovasc Magn Reson. 2012;4:40.

28. Reiter G, Reiter U, Kovacs G, et al. Blood flow vortices along the main pulmonary artery measured with MR imaging for diagnosis of pulmonary hypertension. Radiology. 2015;275(1):71-9.

29. Barker AJ, Roldán-Alzate A, Entezari P, et al. 4D flow assessment of pulmonary artery flow and wall shear stress in adult pulmonary arterial hypertension: results from two institutions. Magn Reson Med. 2015;73(5):1904-13.

30. Freed BH, Collins JD, François CJ, et al. Magnetic resonance and computed tomography imaging for the evaluation of pulmonary hypertension. JACC Cardiovasc Imaging. 2016;9(6):715-32.

31. Jennette JC, Falk RJ, Bacon PA, et al. Revised international chapel hill consensus conference nomenclature of vasculitides. Arthritis Rheum. 2012;65(1):1-11.

Open Access This chapter is licensed under the terms of the Creative Commons Attribution 4.0 International License (http://creativecommons. org/licenses/by/4.0/), which permits use, sharing, adaptation, distribution and reproduction in any medium or format, as long as you give appropriate credit to the original author(s) and the source, provide a link to the Creative Commons license and indicate if changes were made.

The images or other third party material in this chapter are included in the chapter's Creative Commons license, unless indicated otherwise in a credit line to the material. If material is not included in the chapter's Creative Commons license and your intended use is not permitted by statutory regulation or exceeds the permitted use, you will need to obtain permission directly from the copyright holder.

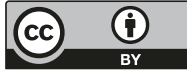

